FORMATION Formation emploi

Revue française de sciences sociales

124 | Octobre-Décembre 2013

Pêle-mêle

\title{
L'entretien de recrutement des gardiens de la paix dans la Police nationale. Paradoxes de l'épreuve et prime à la « compétence interactionnelle »
}

L'entretien de recrutement des gardiens de la paix dans la Police nationale.

Paradoxes de l'épreuve et prime à la " compétence interactionnelle »

French police officers' recruitement interview: paradoxes of the test and bonus

for the skills in the interaction

Das Einstellungsgespräch für die „Gardiens de la paix“ (Ordnungshüter)der

Police Nationale - Prüfungsparadoxe und Bonus für "interaktionelle Kompetenz“

La entrevista de trabajo de los "gardiens de la paix" en la policía francesa.

Paradojas de la prueba y prima a la "competencia interaccional"

\section{Frédéric Gautier}

\section{(2) OpenEdition}

Journals

Édition électronique

URL : http://journals.openedition.org/formationemploi/4088

DOI : $10.4000 /$ formationemploi.4088

ISSN : 2107-0946

Éditeur

La Documentation française

Édition imprimée

Date de publication : 31 décembre 2013

Pagination : 63-86

ISSN : 0759-6340

Référence électronique

Frédéric Gautier, «L'entretien de recrutement des gardiens de la paix dans la Police nationale. Paradoxes de l'épreuve et prime à la « compétence interactionnelle » », Formation emploi [En ligne], 124 | Octobre-Décembre 2013, mis en ligne le 08 janvier 2015, consulté le 30 octobre 2020. URL http://journals.openedition.org/formationemploi/4088 ; DOI : https://doi.org/10.4000/ formationemploi.4088 


\title{
L'entretien de recrutement des gardiens de la paix dans la Police nationale. Paradoxes de l'épreuve et prime à la « compétence interactionnelle »
}

\begin{abstract}
Frédéric Gautier
Professeur agrégé de sciences économiques et sociales dans l'enseignement secondaire. Doctorant (3ème année) en science politique à l'université Lille II - Centre d'Études et de Recherches Administratives, Politiques et Sociales (CERAPS) et doctorant associé au Centre Nantais de Sociologie (CENS - université de Nantes)
\end{abstract}

Résumé

L'entretien de recrutement des gardiens de la paix dans la Police nationale. Paradoxes de l'épreuve et prime à la « compétence interactionnelle »

Larticle analyse une épreuve déterminante du concours de gardien de la paix : l'entretien. Cette épreuve ne s'inscrit pas dans un modèle parfaitement bureaucratique de recrutement. Les jurés disposent en effet d'une marge d'interprétation de leur rôle et expriment des attentes différentes à l'égard des candidats. Le jugement final est pourtant marqué par une forme d'unanimité ; en effet, la réussite à l'épreuve se joue bien plus sur la compétence interactionnelle que sur la conformité aux exigences spécifiques du métier de policier ou sur le degré d'adhésion à l'institution.

Mots clés : recrutement, policier

Abstract

French police officers' recruitement interview : paradoxes of the test and bonus for the skills in the interaction

This article is an analysis of an important part involved in the recruitement of police officers : the interview. This test hardly suits the model of bureaucratic recruitement. The members of the jury have wide range of expectations on interpreting what their role is and what to expect from the candidates. Nevertheless, the final decision is unanimous because the success of the interview is more linked to the skills in the interaction rather than to the ability to comply with the institution or the policemans' job.

Keywords: recruitment, policeman

Journal of Economic Literature: M 51

Traduction : Auteur 
Non sans ironie, Egon Bittner (2001) écrivait : «Le mandat des policiers consiste à affronter tous les problèmes où l'usage de la force risque d'être nécessaire, et si [...] l'on reconnaît quiaccomplir cette tâche de manière socialement utile exige un savoir-faire des plus achevés, alors il serait, semble-t-il, raisonnable de ne choisir pour occuper cette fonction que les plus doués, les plus prometteurs et les plus équilibrés d'entre nous. "L'auteur souligne ainsi certaines spécificités des missions des policiers, en particulier de ceux qui, sur le "terrain ", mettent en œuvre les politiques publiques en matière de sécurité (gardiens de la paix et brigadiers essentiellement ${ }^{1}$ ). Il évoque explicitement la question de leur recrutement, qui semble devoir faire l'objet d'un soin particulier.

Investir le rôle de policier suppose sans doute une certaine adhésion à l'institution, aux normes, valeurs, manières de voir et d'agir qu'elle impose (Lagroye et Offerlé, 2010). Leur incorporation ne peut vraisemblablement pas s'effectuer par l'intermédiaire du seul processus de «(re)socialisation" (Biland, 2010) qui s'y déroule. La socialisation professionnelle, initiée dès l'école de police (Cassan, 2005 ; Moreau de Bellaing, 2006), ne semble pas en effet en mesure de transmettre aux recrues une "culture policière " monolithique (Gorgeon, 1996 ; Monjardet et Gorgeon, 1993 ; Monjardet, 1996)² qui déterminerait entièrement les modalités de l'agir policier. Il peut alors apparaître nécessaire que les futures recrues aient déjà intériorisé certaines dispositions (un rapport à la Loi fortement teinté de légalisme, une stabilité émotionnelle, une aversion limitée au risque par exemple) ou qu'à tout le moins, elles soient aptes à faire preuve d'une "forme de docilité " (Lagroye et Offerlé, 2010), susceptible de faciliter le processus d'intégration dans l'institution. Aussi est-il pertinent d'interroger l'hypothèse selon laquelle cette dernière accorde " une attention particulière " (Biland, 2010) au choix des impétrants.

Bien que le recours à des personnels contractuels (les Adjoints de Sécurité ou ADS) soit largement utilisé par l'administration de la Police nationale, le concours demeure la seule voie d'accès aux emplois statutaires et, en ce sens, il constitue un élément essentiel du dispositif par le biais duquel elle recrute ses agents. Dès lors, on peut s'interroger sur ce que le concours révèle des attentes de l'institution à l'égard des membres de son personnel, sur ce qu'il dit des dispositions et propriétés dont elle souhaite voir dotés ses agents, ainsi qu'en creux, sur celles qu'elle juge incompatibles avec les missions qui leur seront confiées

Certains candidats peuvent choisir une épreuve qui leur permet d'être évalués sur des connaissances en partie liées à l'exercice du métier de policier ; toutefois, les épreuves d'admissibilité du concours ${ }^{3}$ sont, dans l'ensemble, très semblables, dans leurs formes comme

1. Les brigadiers sont tous d'anciens gardiens promus.

2. Ces différents travaux contribuent à mettre en question la thèse de l'existence d'une sous-culture policière unifiée et homogène, constitutive d'une "personnalité de travail " commune à tous les policiers, défendue par certains sociologues anglo-saxons. Voir notamment Becker, 1985 ; Skolnick, 1966 ; Van Maanen, 1973. 3. Ces épreuves sélectionnent les candidats sur la base de compétences rédactionnelles et cognitives, ce qui tend à handicaper les candidats les moins dotés en capital scolaire et plus généralement en capital culturel, et rend difficile l'accès à la fonction publique pour les jeunes issus des milieux les plus défavorisés. Il faut cepen- 
dans leurs modes d'évaluation, à celles qui figurent au programme d'autres concours de la fonction publique de catégorie identique (voir encadré 1).

\section{Encadré 1}

\section{Les épreuves du concours}

Il existe trois voies d'accès au statut de gardien de la paix : le concours externe (ouvert aux titulaires du baccalauréat ou d'un diplôme équivalent), le concours interne ou second concours (ouvert aux adjoints de sécurité sans condition de diplôme) et le concours dit « réservé ", essentiellement ouvert aux militaires.

Les candidats aux concours externe et interne sont soumis aux mêmes épreuves écrites d'admissibilité. II s'agit :

- d'un « questionnaire portant sur les fondamentaux scolaires en orthographe et grammaire courantes ainsi que sur la capacité à résoudre des calculs arithmétiques et algébriques simples » (coefficient 1)

ET

- d'un « questionnaire portant sur les connaissances générales en rapport avec les événements qui font l'actualité, le cadre institutionnel politique français et européen et les règles du comportement citoyen », dit aussi « questionnaire à réponse unique de culture générale » (coef. 2)

OU (au choix des candidats)

- d'un « questionnaire portant sur le programme du baccalauréat professionnel spécialité sécurité - prévention en vigueur l'année d'ouverture du concours». Ce dernier comporte, outre des questions de " culture générale » similaires à celles qui figurent dans le questionnaire du premier type, une proportion élevée de questions centrées sur les métiers auxquels prépare le baccalauréat mentionné (policier ou ADS, gendarme, pompier professionnel ou militaire, agent de sécurité....).

ET

- d'une « étude d'un texte permettant de vérifier la prise d'informations et l'analyse de celles-ci, sous forme de courtes questions, ainsi que la production d'un écrit en rapport avec la problématique posée ». (coef. 3)

Pendant la phase d'admissibilité, les candidats subissent également des tests psychotechniques, non notés mais utilisés au moment de l'épreuve d'entretien (coef. 4), lors de la phase d'admission, qui se compose en outre d'une épreuve sportive (coef. 3) et d'une épreuve de langue étrangère (coef. 1).

Les candidats au concours réservé sont d'abord soumis à une pré-sélection sur la base de tests psychotechniques et d'une épreuve sportive. En cas de succès, ils passent la même épreuve d'entretien que les autres candidats.

dant noter que le degré de fermeture du concours aux moins dotés scolairement et culturellement dépend en dernière instance du rapport postes à pourvoir/candidats et de la note en-dessous de laquelle l'administration décide de ne plus admettre de candidats. 
Moins répandue ${ }^{4}$, l'existence d'une épreuve sportive témoigne de la volonté de l'institution de faire de la possession de certaines ressources corporelles (endurance, vitesse, agilité...) une condition d'accès à la fonction policière. Mais c'est à travers l'épreuve d' " entretien avec le jury permettant d'apprécier les qualités de réflexion et les connaissances du candidat ainsi que son aptitude et sa motivation à exercer l'emploi postulé " que l'institution est susceptible de révéler le mieux ses attentes spécifiques au regard des particularités des missions policières. Quels enseignements peut-on alors tirer d'une sociologie de cette épreuve ? Quelles en sont les modalités pratiques? Quels principes et catégories de jugement mobilise-t-elle ? Telles sont les questions à laquelle tente de répondre cet article, à partir d'une enquête mobilisant observation ethnographique et entretiens (voir encadré 2).

Dans une première partie, nous montrerons que l'entretien apparait, aux yeux des jurés interrogés, comme l'élément central du dispositif de recrutement, seul susceptible de déceler ,chez les candidats, les qualités spécifiques nécessaires à l'exercice de la fonction de gardien de la paix, alors même qu'il n'existe pas de consensus sur la définition de ces qualités. Nos observations permettront ensuite de montrer que la manière dont se déroule l'épreuve oblige à rompre avec la vision d'un mode de recrutement parfaitement rationalisé et bureaucratisé. Dans la troisième partie, nous analyserons les principes et catégories de jugement mis en oeuvre lors de l'évaluation. Il apparaîtra que, malgré ou par-delà la diversité des attentes qu'expriment les membres des jurys à l'égard des futures recrues, une forme d'unanimisme prévaut lorsqu'il s'agit d'apprécier la prestation d'un candidat. L'explication de cette situation paradoxale réside dans le fait que la réussite à l'épreuve se joue moins sur la conformité des futures recrues à un certain nombre d'attentes directement liées aux particularités de la fonction que sur l'habileté des candidats dans l'interaction avec le jury.

\section{Encadré 2}

\section{Méthode d'enquête}

Alors que l'institution policière est parfois présentée comme particulièrement fermée aux investigations scientifiques (Monjardet, 2005 ; Loubet del Bayle, 2006), notre enquête, dès lors qu'elle fut autorisée par la Direction centrale de la police nationale, n'a pas rencontré de réticences notables. Si la possibilité d'assister en tant qu'observateur aux épreuves d'un concours de la fonction publique est un droit garanti à tout citoyen, nous n'avons jamais eu à nous prévaloir de ce droit (qui ne s'applique d'ailleurs pas aux délibérations) pour accéder à notre terrain. L'accueil qui nous a été réservé par les différents membres de l'institution rencontrés, leur disponibilité et la liberté de parole (1) qu'ils ont manifestée lors des entretiens doivent sans doute beaucoup à deux « alliés » (un capitaine de police dans le premier centre et un agent administratif dans le second) qui, intéressés par notre projet de recherche (2), ont su nous introduire auprès de leurs collègues et faciliter nos démarches au sein de l'institution

4. Elle figure néanmoins au programme d'autres concours de la fonction publique, ceux de contrôleur des douanes ou de professeur d'éducation physique et sportive par exemple, ainsi qu'au concours d'entrée à l'Ecole nationale d'administration. 


\section{Suite encadré 2}

Le matériau empirique mobilisé dans cet article est issu de séries d'observations de l'épreuve d'entretien des trois concours (externe, interne et réservé) réalisées dans deux centres d'examen différents, lors des épreuves de la deuxième session annuelle de 2007 et de la première session de 2008 ( 25 prestations et délibérations observées, soit cinq jurys différents) pour l'un, lors de la session unique de 2011 (12 prestations, trois jurys différents) pour l'autre.

Si elle n'avait pas pour objectif de vérifier une hypothèse énoncée a priori (dans le cas contraire, le chercheur s'expose au risque de ne trouver que ce qu'il cherche), l'observation était néanmoins orientée par un questionnement relatif à l'homogénéité ou l'hétérogénéité des pratiques des jurés. II s'agissait donc de repérer autant ce qui, d'une prestation à l'autre, d'un évaluateur à l'autre, d'un jury à l'autre, se retrouvait de manière régulière, que ce qui pouvait apparaître singulier, pour, ensuite, interroger les facteurs ou les conditions sociales produisant l'homogénéité versus l'hétérogénéité (3).

Dans le premier centre d'examen, nous avons cumulé les prestations observées jusqu'au moment où il nous est apparu que l'observation d'une prestation de plus (avec un même jury d'abord, avec l'ensemble des jurys ensuite) aurait eu un « rendement » faible (4). En 2011, nous avons pu mener une autre série d'observations dans un deuxième centre, situé dans une autre région (5). Cette forme de " retour sur le terrain » a permis de questionner le degré de " saturation » atteint par la première série d'observations et le caractère généralisable des données obtenues. Elle a ainsi montré que les pratiques régulières observées dans un centre l'étaient également dans l'autre et semblaient donc indépendantes des contextes locaux.

Aux observations s'ajoutent des entretiens avec des jurés (6). Dans chaque jury siègent un fonctionnaire du corps de conception et de direction de la Police nationale, un fonctionnaire du corps de commandement et d'encadrement, un fonctionnaire du corps de maîtrise et d'application et un psychologue, souvent extérieur à l'institution et titulaire pour l'occasion d'un statut de vacataire. Le choix des enquêtés $(n=12)$ tient compte de cette diversité de statuts. Les entretiens de type semi-directif (d'une durée comprise entre $1 \mathrm{~h}$ et $2 \mathrm{~h}$ ), enregistrés, portent sur les pratiques en tant que juré, sur la manière dont ces pratiques sont perçues, et sur les trajectoires sociales et professionnelles des enquêtés. Des discussions plus informelles viennent par ailleurs utilement compléter ces matériaux.

Ce travail entend s'inscrire dans une sociologie attentive à la fois à l'action (de recruter, de sélectionner, de juger) et aux acteurs (recruteurs et candidats) (Voir par exemple Cartier, 2001 ; Eymeri, 2001 ; Réau, 2009 ; Darmon, 2012). II cherche à articuler un premier type d'approche, inspiré de la sociologie pragmatique et de l'ethnométhodologie (Voir par exemple Eymard-Duvernay et Marchal, 1997), qui invite à une étude fine des actions et interactions composant l'« acte d'engager » et un second type d'inspiration bourdieusienne qui, s'il peut conduire à laisser à l'état de boîte noire le processus de recrutement, permet d'éviter de traiter la rencontre entre candidats et recruteurs comme " un empire dans un empire » (Bourdieu, 1982), en montrant tout ce que la production du jugement doit aux positions et aux dispositions des protagonistes.

(1) Les propos tenus, parfois ouvertement critiques à l'égard de collègues ou de l'administration de tutelle, témoignent du fait que, dans la plupart des cas, nous ne nous sommes pas heurtés une « langue de bois ». Dire cela, ce n'est pas tomber dans l'illusion naïve selon laquelle l'entretien permet de recueillir un discours " vrai », d'abord parce que le discours, loin d'être un " déjà-là », en attente d'être collecté par le chercheur, est produit pour et au cours de l'entretien ; ensuite, parce que la production de ce discours est tributaire des conditions de l'entretien luimême, incluant notamment les dispositions de l'enquêté comme celles de l'enquêteur. 


\section{Suite et fin encadré 2}

(2) Les faits que tous deux soient diplômés de l'enseignement supérieur, que le second ait été enseignant dans le secondaire, ne sont sans doute pas étrangers aux bonnes dispositions qu'ils ont montrées à l'égard de ce projet.

(3) Sauf à prétendre « tout voir » de chacune des situations d'entretien et à multiplier à l'infini les situations observées, il est, par définition, impossible d'« épuiser » la singularité, i.e. de rendre compte de manière exhaustive de l'hétérogénéité et de la diversité des pratiques.

(4) Le rendement marginal de l'observation n'est jamais nul. La détermination du moment où il est suffisamment faible pour que le terrain puisse être quitté " sans regret » est difficile et le choix fait par le chercheur toujours susceptible d'être contesté (Arborio et Fournier, 2008). C'est en tout cas moins une logique de représentativité ou d'exhaustivité qui doit présider à ce choix qu'une logique de saturation (Barbot, 2010).

(5) La préservation de l'anonymat de nos enquêtés et le " contrat » passé avec eux nous contraignent à limiter la présentation des données de contexte.

(6) Dans le cadre du projet de recherche plus global dont est issu cet article, nous menons également une campagne d'entretiens auprès de candidats (notamment sur leurs motivations pour entrer dans la police et le travail fourni pour se préparer au concours). Nous avons également rencontré des organisateurs du concours, afin d'obtenir des informations sur les modalités des épreuves et de composition des jurys.

\section{Le discours des acteurs : croyance partagée en l'efficacité de l'épreuve, mais diversité des attentes}

Les épreuves d'admissibilité, l'épreuve de langue étrangère ou l'épreuve sportive constituent, aux yeux des membres de l'institution, des étapes nécessaires mais insuffisantes du dispositif de sélection. Elles auraient pour fonction essentielle d'opérer un premier tri grossier parmi les candidats, en éliminant ceux qui ne peuvent attester d'un niveau jugé minimum dans des champs de compétence généraux et transversaux (maîtrise de l'orthographe et de la syntaxe notamment, aptitudes physiques). Ces compétences non spécifiques sont néanmoins considérées comme indispensables. Elles sont en effet perçues comme susceptibles d'influer sur l'efficacité de l'activité policière la plus quotidienne (rédaction de procès-verbaux par exemple).

Pour autant, ces épreuves ne suffiraient pas à garantir la conformité des candidats à toutes les exigences spécifiques que la fonction impose. Ce rôle est dévolu à l'épreuve d'entretien. Outre qu'elle est dotée d'un coefficient important qui lui confere objectivement un rôle déterminant dans la sélection, elle est - du fait qu'elle place en situation de co-présence un candidat et des examinateurs dont l'aptitude et la légitimité à juger semblent présumées ${ }^{5}$ -

5. Deux registres différents sotn mobilisés par nos enquêtés pour (se) convaincre de l'aptitude à juger des examinateurs. Pour les jurés policiers, il existe une présomption de légitimité déduite de leur supposée 
très généralement perçue comme un instrument pertinent et efficace du recrutement. Plusieurs arguments sont convoqués par nos enquêtés pour étayer cette croyance. D’abord, l'expertise reconnue par les jurés policiers au psychologue membre du jury doit permettre d'éliminer les candidats qui présenteraient des traits psychologiques (état dépressif, instabilité émotionnelle, impulsivité...) réputés incompatibles avec la fonction policière. C’est ainsi que les policiers interrogés considèrent les tests psychotechniques, leur interprétation par le psychologue et la capacité présumée de celui-ci à affiner son jugement lors de l'entretien comme un ensemble de moyens fiables pour limiter, sinon empêcher, le recrutement de candidats jugés indésirables.

Outre cette fonction d'élimination, l'entretien doit permettre de déceler, chez les candidats, des qualités jugées indispensables. Le discours des examinateurs met invariablement - et parfois exclusivement - en avant deux attentes fondamentales à l'égard des candidats : ceuxci doivent être dotés d'une "culture générale " suffisante et animés d'une " motivation » bien réelle pour la fonction.

\subsection{Quelle culture générale ?}

Les entretiens montrent toutefois que l'usage de termes identiques masque la variabilité des significations qui leur sont attribuées. Ainsi, chez les plus gradés et les plus titrés scolairement, la définition de la " culture générale » renvoie parfois à une simple forme d'ouverture sur le monde ou même de sens pratique du placement dans l'espace social, comme si l'on ne pouvait pas attendre plus d'un gardien de la paix. D'autres, souvent moins gradés, l'utilisent dans une acception plus habituelle, renvoyant à la maîtrise de connaissances relevant de la culture légitime et scolaire, comme le montrent ces trois extraits d'entretien :

"Alors, ça ne veut pas dire qu'on attend des gens qu'ils soient cultivés au point d'aller s'entretenirje ne sais pas moi - avec Daniel Cohn-Bendit et Xavier Darcos sur l'éducation. Ça, on n'en a rien à foutre hein. [...] Il faut que le gardien de la paix soit capable d'appréhender le milieu dans lequel il intervient. C'est-à-dire, le milieu, c'est la société, dans ses... dans tous ses milieux, qu'il sache à qui il a affaire. [...] Ça veut dire aussi qu'on s’intéresse à ce qui se passe dans la vie et dans les médias. [...] On ne leur demande pas d'être des analystes et de livrer leur analyse sur FranceInfo le lendemain, au cours d'une chronique, ce n'est pas ça. Ce qu'on demande, c'est qu'ils soient au courant, qu'ils sintéressent un petit peu au monde dans lequel ils vivent. C'est tout! Voilà ! Qu'ils soient so-cia-li-sés! C'est une marque de socialisation. Voilà. C'est tout. "Entretien avec une lieutenant, titulaire d'un DESS (diplôme d'études supérieures spécialisées)

"Alors parler de culture générale... Le concours de gardien de la paix, ce n'est pas un concours... ce n'est pas l'ENA, ni Polytechnique, hein. C'est un concours qui est niveau bac donc c'est un concours qui est, je dirais, assez basique dans la fonction publique en général. Moi ce que j’at-

connaissance du terrain. C'est au contraire leur situation d'extériorité et la reconnaissance de la validité des connaissances scientifiques qu'ils mobilisent qui confèrent leur légitimité aux psychologues. 
tends, c'est qu'au niveau de la personnalité et de la culture générale - je mets ça ensemble jattends des standards minimum. Minimum. Et quand je suis en-dessous du minimum, pour moi c'est éliminatoire. Le minimum, c'est quoi ? Ben, c'est quelqu'un qui soit un minimum curieux, qui me semble un minimum curieux, capable de communiquer - la communication, c'est très important. " Entretien avec un commissaire principal, titulaire d'un diplôme de troisième cycle en droit

"On a cette technique, par exemple, lorsqu'on leur demande dès le début - ben vous étiez là-les passions. [...] On leur demande les passions. Par exemple, souvent, ce qu'ils ont tendance à nous dire, c'est le sport et donc les arts martiaux et la lecture! Comme ça, on a euh... un corps sain dans un esprit sain! Et ce qui est intéressant, c'est lorsqu'on laisse passer à peu près 12-13 minutes et qu'on leur demande qui était Étienne de la Boétie ou Montaigne et que là, ce n'est pas trop... ils ne savent pas trop de qui ils sont en train de parler. "Entretien avec un brigadier-chef, bachelier)

\subsection{Quelle « motivation »?}

Dans le même ordre d'idées, les conceptions de la "motivation ", des «bonnes raisons " de passer le concours, c'est-à-dire des justifications recevables, sont diverses.

Ainsi, pour certains jurés, il est légitime de vouloir entrer dans la police pour la seule raison qu'elle peut fournir un cadre propice à l'exercice d'une activité que le candidat apprécie (rouler à moto, s'occuper de chiens...) quand bien même elle n'aurait rien de spécifiquement " policier ". Entretenir un rapport que l'on pourrait dire instrumental à l'administration dans laquelle le candidat prétend entrer n'est donc pas disqualifiant mais apparaît même comme une forme de garantie que la recrue trouvera un intérêt dans l'exercice de sa fonction et non dans le seul statut que celle-ci offre. La figurerepoussoir qui se dessine en creux ici est celle du "fonctionnaire planqué ».

Pour d'autres examinateurs, il semble impératif que le candidat réfère explicitement ses motivations à des missions spécifiquement - sinon exclusivement - policières, desquelles sont formellement exclues les actions qui pourraient s'apparenter au travail social. Et c'est ici la figure de l' " assistante sociale " qui est rejetée.

Cette hétérogénéité dans les attentes des examinateurs - que laissent voir les extraits d'entretien ci-dessous - révélatrice de conceptions diverses du métier policier, doit autant aux différences de position et de trajectoire dans l'institution qu'aux expériences socialisatrices en dehors de l'institution. C'est ainsi que les attentes d'un brigadier ont toutes les chances d'être différentes de celles d'un commissaire. Pour autant, il est impossible d'associer un type d'attentes à une position dans l'institution car, à ce clivage vertical s'ajoute un clivage horizontal. Les différences entre deux membres du 
même corps peuvent être tout aussi sensibles et il faut sans doute en chercher l'origine dans les dispositions diverses dont ils sont porteurs.

"Il faut que quelque chose le passionne dans le métier de gardien de la paix. Alors, ça peut être le goût du contact avec le public, ça peut être le goût du droit ou, au contraire, le gồt de la moto parce que vraiment il veut être motard et il fera tout pour y arriver. Ou bien le goût des animaux, des chiens par exemple, il veut être maître-chien... Enfin, il faut qu'il nous montre une passion quelconque. [...] Parce que si le jeune arrive déjà en marche arrière, à vingt ans, au boulot, c'est l'horreur, quoi. Vous comprenez?" Entretien avec un commissaire de police

"Moi, quand je juge la motivation d'un candidat, je me réfere aux missions de la Police nationale. [...] On ne rentre pas dans la police pour discuter avec les gens, pour tailler le bout de gras avec la boulangère, ni pour être sympa avec tout le monde. [...] Est-ce qu'il recherche cette mission-là ou est-ce qu'il ne cherche pas une mission d'assistante sociale ou d'éducateur spécialisé, ce qui est complètement différent? [...] J'attends d'un candidat qu'il me dise effectivement pourquoi il veut rentrer dans la police et que ce qu'il dise corresponde à une mission que moi, je me représente. " Entretien avec un commissaire principal de police

La prise en compte de cette diversité a une conséquence fondamentale dans la manière dont le concours peut être pensé sociologiquement ; elle soulève ensuite une question importante. Premièrement, elle oblige à renoncer à l'idée qu'à travers le concours, c'est l'institution, pensée comme totalité unifiée, qui révèle et exprime ses attentes à l'égard de ses recrues. Elle nous éloigne de la conception wébérienne du concours comme dispositif parfaitement objectif et rationnel, en un mot bureaucratique, tant y entre, semble-t-il, la subjectivité des acteurs chargés de sa mise en oeuvre. La fiction d'un concours bureaucratique est d'ailleurs soulignée par l'étude des pratiques auxquelles il donne lieu (cf. 2ème partie).

Deuxièmement, il faut se demander comment ces attentes différenciées, telles qu'elles s'expriment dans les discours des examinateurs, informent effectivement leurs pratiques, principes et catégories de jugement sur les candidats ( $c f$. 3ème partie).

\section{Les « pratiques » de l'entretien : une standardisation partielle}

Si leur généralisation dans la fonction publique a partie liée avec les processus de rationalisation des activités sociales et de bureaucratisation (Weber 1995), les concours, encadrés dans leurs objectifs et modalités d'organisation comme d'évaluation par un ensemble de textes réglementaires, ne peuvent, en pratique, être assimilés à une sélection parfaitement bureaucratisée, opérant sur des critères précisément définis, rationnels et objectivables. C'est particulièrement clair pour les épreuves d'entretien qui placent les candidats face aux examinateurs, les uns comme les autres interprétant leur rôle respectif lestés de leurs dispositions personnelles. Avant d'analyser la production du jugement proprement dit, 
nous montrerons, dans cette deuxième partie, comment les jurés du concours de gardien de la paix s'approprient l'épreuve et la mettent en oeuvre en interprétant les textes officiels qui la définissent. La définition officielle de l'épreuve se borne à en préciser les objectifs mais reste silencieuse sur les conditions concrètes de son déroulement. Les jurés disent par ailleurs ne pas recevoir de consignes précises de leur administration quant aux critères d'évaluation à appliquer. Dans l'ensemble, pourtant, les jurys procèdent de manière peu différente les uns des autres : l'organisation générale (dans le temps et l'espace notamment) est la même pour tous et, surtout, de nombreuses questions reviennent très fréquemment, comme si le répertoire disponible était borné et comportait des " passages obligés ». Cette relative homogénéité des pratiques tient avant tout au mode d'apprentissage du rôle de juré, qui se fait pour l'essentiel "sur le tas ", par l'observation des pratiques des autres membres du jury. Si le principe d'une formation dispensée aux jurés ${ }^{6}$ semble en voie de généralisation, ses modalités d'organisation (deux journées, parfois plusieurs mois avant les épreuves) obligent à relativiser son influence sur les pratiques. En outre, certains de nos enquêtés ne l'avaient pas (encore) reçue au moment de l'enquête et ils en contestent parfois l'intérêt, comme l'illustre le premier extrait d'entretien ci-dessous :

"Est-ce que vous recevez des consignes précises justement quant au déroulement de l'entretien, est-ce que vous êtes briefé avant, est-ce que vous avez reçu une formation?"

"Aucune formation. Pas de formation, pas de consigne. [] Aucune démarche. En fait, on part du principe - et je pense que c'est un bon principe - que nous [...] sommes membres du corps de conception et de direction de la Police nationale, on est directeur de service donc... on est chef de service donc voilà, on part du principe que les attentes qu'on a d'un gardien de la paix ou d'un candidat au poste de gardien de la paix, on n'a pas à nous expliquer ce qu'on attend. Moi je sais exactement ce qu'on attend d'un gardien de la paix, je sais exactement quel profil je recherche. Enfin voilà, la formation ne nous semblerait pas du tout bienvenue, quoi. Le métier de gardien de la paix, c'est un métier qu'on connaît puisquion dirige les gardiens de la paix toute la journée, donc on recrute des gens qu'on est amené à diriger plus tard, des futurs collaborateurs, grosso modo. " Entretien avec un commissaire principal

"Est-ce que vous avez suivi une formation particulière pour devenir membre du jury?"

"Non. Non. Je dirais que souvent on se renseigne par rapport aux anciens membres du jury pour savoir comment ils travaillent. [...] En règle générale, quand on est membre du jury, celui qui n'a jamais fait d'entretien de recrutement, les deux-trois premiers, il essaye de voir comment les autres membres du jury travaillent. " Entretien avec un brigadier-chef

Pour l'essentiel, les nouveaux venus dans les jurys composent donc avec le dispositif tel qu'il se présente et s'impose à eux, en alignant leur comportement sur celui de leurs pairs. Ainsi, les manières d'agir, le contenu et la forme des interrogations par exemple, se transmettent,

6. Cette formation a pour objectif d'expliciter les objectifs de l'épreuve et de donner aux jurés des conseils quant à la manière dont elle peut être conduite. 
se répètent, et finissent par constituer un fonds commun de pratiques, mobilisé par tous et qui reste en grande partie impensé. On peut cependant, par-delà l'homogénéité dans la conduite globale du dispositif, repérer des variations significatives dans les pratiques, d'un jury à l'autre comme d'un examinateur à l'autre.

C'est d'abord du côté de ce que l'on pourrait appeler la "mise en scène » que s'observent des différences importantes. Si la disposition du " décor » est immuable, chaque jury apporte en effet une tonalité, une " ambiance " particulières à la "représentation". Dans certains cas, les candidats sont accueillis d'une manière presque cordiale, se voient présenter clairement les objectifs de l'entretien et sont parfois invités à " se détendre " alors que, dans d'autres, au contraire, l'accueil se veut très froid et impersonnel. Les questions peuvent être posées de manière abrupte voire agressive, les examinateurs interrogés invoquant la nécessité d'éprouver la maîtrise de soi des candidats. D'autres semblent se refuser à employer ces techniques de déstabilisation. D'une manière générale, il semble que, dans chaque jury, le commissaire contribue fortement au choix de la " mise en scène ". C'est lui qui donne la tonalité générale de l'entretien, les autres examinateurs semblant se contenter de composer avec ce parti pris «scénique ». Les «clivages structurels» (Goffman, 1973) apparaissent ici relativement prégnants, comme lors d'épreuves du même type dans d'autres concours de recrutement (Cartier, 2001).

Par ailleurs, la représentation n'est jamais totalement "écrite " à l'avance. Il arrive donc que des interventions sortent du répertoire " classique ", c'est-à-dire du " fonds commun », comme en attestent ces extraits de nos notes d'observation :

Le candidat (père : technicien de maintenance, mère au foyer) est un ADS qui se présente au concours pour la 8ème fois. Tout dans son hexis corporelle et sa manière de s'exprimer (le candidat commet à plusieurs reprises des "fautes " de syntaxe, emploie des termes "familiers") trahit son origine populaire et rurale. Le commissaire lui demande en levant le menton "Et qu'est-ce vous faites là-bas, à $A .{ }^{7}$, vous sortez de temps en temps? Hein, votre grande passion à A., c'est quoi ?». Le candidat raconte ensuite une interpellation à laquelle il a participé. En courant, il a réussi à empêcher la fuite d'un contrevenant. Le commissaire conclut : "Vous êtes le Forrest Gump d'A., vous, hein! " (Concours interne)

Le candidat est ADS. La consonance de son patronyme laisse supposer une ascendance maghrébine. Il est le seul candidat que nous rencontrons à qui il est demandé de préciser l'origine de sa famille. Le commissaire lui demande quelle équipe il supporte lors des matches de foot opposant la France et le Maroc, s'il se sent 100 \% Français. Le

7. A. est une petite ville située dans le même département que la métropole locale où se déroulent les épreuves. De par ses caractéristiques (relatif éloignement par rapport à la métropole, population d'origine populaire et rurale...), A. est souvent la cible de quolibets de certains habitants de la métropole.

8. Allusion à un film américain de 1994 qui met en scène un homme simple d'esprit, connu pour son aptitude à la course. 
candidat hésite puis répond qu’il est «à $90 \%$ Français ». Il est également interrogé sur les réactions de ses "copains", lorsqu'ils apprendront qu'il est "flic ». Il est le seul qui se voit proposer une " mise en situation " impliquant son petit frère dans un trafic de stupéfiants. Une fois l'entretien terminé, la psychologue manifeste son désaccord sur l'orientation donnée par le commissaire au questionnement. Elle contestera aussi les remarques faites lors des délibérations. (Concours interne)

Le candidat (ADS), dont la manière de parler témoigne de son origine populaire, est soumis à un flot de questions se succédant à un rythme très rapide sur l'organisation de la police, puis le brigadier-chef (femme) lui demande : "La discrimination positive, ça vous dit quelque chose?". Devant l'absence de réponse, l'examinatrice soupire en levant les yeux au ciel puis dit, avec un geste significatif: "Alors à part les chiens et le foot... ». Cette examinatrice est, au sein du jury, celle qui pose le plus de questions de "culture générale ", au point que nous nous demandons si elle ne se livre pas à une stratégie de présentation de soi destinée aux autres membres du jury - au sein duquel elle est en position dominée (elle est la moins gradée et une femme, qui plus est) - visant à attester de sa légitimité à être juré. (Concours interne)

Il apparaît alors que des questions posées ou des remarques adressées aux candidats trahissent certaines dispositions des examinateurs, un peu à la manière de "lapsus " révélateurs de leur identité sociale et parfois empreints de formes de mépris de classe, de sexisme ou teintés de stéréotypes culturalistes. On peut également repérer que les questions ou remarques adressées aux candidats semblent parfois moins destinées à mettre ces derniers à l'épreuve qu'à proposer aux autres examinateurs une présentation avantageuse de soi, en manifestant, par exemple, une habileté discursive, une maitrise de la culture la plus légitime, ou une connaissance des "réalités " du " terrain ".

Partiellement standardisée seulement, l'épreuve d'entretien ne se laisse donc pas totalement circonscrire par les textes officiels censés en définir les modalités : elle échappe en partie au contrôle de l'institution et n'a que peu à voir avec une stricte rationalité bureaucratique. Qu'en est-il de la production du jugement ? Sur quels critères repose l'évaluation des candidats et selon quels principes aboutit-elle à la production de l'appréciation et de la note dont ils se verront gratifiés ?

\section{Ce qui est (vraiment) « mis à l'épreuve »: 5 une prime à la compétence interactionnelle ?}

L'observation des délibérations révèle une situation paradoxale : c'est une forme d'unanimisme qui prévaut quand il s'agit de commenter et d'apprécier les prestations des candidats. Malgré la diversité des attentes que mettent en évidence les entretiens, la production du jugement final (i.e. l'appréciation portée sur chaque candidat et la note donnée) ne s'apparente pas à un travail d'agrégation de préférences hétérogènes. Elle n'est pas le 
résultat d'une négociation visant à opérer la synthèse d'avis divergents et potentiellement incommensurables car mobilisant, consciemment ou inconsciemment, des registres différents (Boltanski et Thévenot, 1991).

La délibération qui suit chaque entretien ne dure que quelques minutes. Elle prend la forme d'échanges brefs, "sans cérémonie ", au cours desquels chacun prend la parole quand il le souhaite, sans respect particulier d'une quelconque préséance hiérarchique, d'une manière parfois très relâchée. La discussion est émaillée de considérations étrangères à la production du jugement (sur la société, la police...). S'il est indéniable que le commissaire est maître du déroulement de l'entretien, son influence ne se fait guère sentir au moment de la formulation du jugement, pendant lequel tout indique que les clivages structurels sont atténués. Aucun des examinateurs ne propose une argumentation élaborée explicitant les principes qui fondent son jugement. Chacun se contente de formules lapidaires, comprises par tous et ne donnant lieu à aucune mise en question. Surtout, il n'y a aucune divergence notable entre les opinions émises sur chaque candidat, qui se voit rapidement classé dans une des catégories le plus souvent utilisées par les évaluateurs et qui synthétisent le jugement : les «bons », les "moyens », les « mous"?.

Cette univocité du jugement parait contradictoire avec l'existence d'attentes différenciées selon les évaluateurs. Pour en rendre compte, c'est la nature même de l'épreuve (et de ce qui est mis à l'épreuve) qu'il nous faut interroger. Nous pouvons alors montrer qu'elle n'est pas tout à fait conforme à ce que les examinateurs disent en attendre et vouloir en faire.

\section{1. Éprouver les connaissances et la « motivation » ?}

Nos observations montrent d'abord que l'essentiel ne se joue pas sur le terrain cognitif. Les connaissances (de type professionnel ou scolaire) ne sont en effet pas décisives dans la sélection des recrues, soit parce qu'elles sont peu discriminantes (beaucoup de candidats sont en mesure de les mobiliser), soit parce qu'elles n'apparaissent pas toujours indispensables.

Ainsi, les questions relatives au fonctionnement et à l'organisation générale de l'institution posées aux candidats ${ }^{10}$ n'ont pas pour but d'opérer une sélection entre les déten-

9. L’usage fréquent de ce terme pour qualifier les candidats obtenant une note faible mériterait une analyse plus détaillée que ne le permet le cadre de cet article. La "mollesse » des candidats renvoie essentiellment à leur manque supposé de dynamisme et à leur incapacité présumée à prendre des initiatives. Le terme condense donc un certain nombre de "défauts ", vus comme incompatibles avec les exigences du métier de gardien de la paix.

10. Tous les candidats sont soumis à ce type de questions mais pour les candidats internes, elles sont plus précises et plus techniques et se réferent à leur expérience professionnelle. 
teurs d'une connaissance rare, dont la possession serait le fruit d'un lent et long travail de "bachotage ", et les autres.

Pour tous les examinateurs, il s'agit plutôt de « tester la motivation » des impétrants, selon un principe affiché qui veut qu'un candidat "motivé » se soit donné la peine de se préparer à ce type de questions. De fait, parmi ceux que nous avons observés, les (rares) candidats qui n'étaient visiblement pas dans ce cas ont tous obtenu une note rendant leur recrutement hautement improbable. Reste que de nombreux candidats, particulièrement ceux qui n'en sont pas à leur première tentative ou qui ont pris le soin de se préparer au concours, parviennent à mobiliser ces connaissances, dont la détention apparaît dès lors comme indispensable mais insuffisante. Une sélection s'opère donc encore parmi ceux-là.

Les questions dites de " culture générale " et de " connaissance de l'actualité " ne revêtent, quant à elles, qu'une importance mineure. Plus exactement, une faible réussite en la matière ne handicape que les candidats qui font, pour d'autres raisons ( $c f$. le point 3.3), l'objet d'une appréciation négative. Pour les autres, elle semble n'avoir tout simplement aucune incidence.

Par ailleurs, si l'entretien permet d'éliminer ceux qui n'ont qu'une idée très vague de ce qui les attend et semblent se présenter au concours sans l'avoir préparé, on ne peut le considérer comme un contrôle de motivation qui opérerait une sélection des candidats sur la base de la force et de la légitimité de leurs raisons - effectives et non seulement déclarées - de postuler. D'abord parce que leur discours est, dans la plupart des cas, pré-construit pour être conforme aux attentes supposées du jury, ensuite parce que parmi les (nombreux) candidats qui invoquent de «bonnes » raisons (et tiennent alors en réalité des propos très semblables), beaucoup obtiennent une note moyenne ou faible. Dès lors, il est possible d'affirmer que si la capacité à produire un discours de justification recevable est nécessaire (et son absence rédhibitoire), elle ne suffit pas à assurer aux candidats l'obtention d'une note garantissant leur recrutement. C'est donc que la sélection ne s'opère pas exclusivement, loin s'en faut, sur la question de la « motivation ».

\subsection{Dépister les « bonnes » (pré-)dispositions ?}

On pourrait enfin s'attendre à ce que l'institution s'efforce de se doter de recrues porteuses de dispositions particulières qui augureraient d'une " aptitude à exercer l'emploi postulé ". Il est donc frappant de constater que l'épreuve d'entretien ne permet guère de déceler l'aptitude à obéir aux ordres et à se plier aux exigences de la discipline, la soumission et la loyauté envers la hiérarchie, des recrues potentielles.

Nos observations montrent que ces qualités sont présumées dès lors que le candidat " joue le jeu ", c'est-à-dire participe à l'entretien en respectant les règles implicites qui régissent ce type de situation : l'usage de marques de politesse, la posture, la tenue vestimentaire doivent témoigner d'une forme de déférence à l'égard des membres du jury. Mais ces règles sont 
communes à la majorité des entretiens d'embauche ou des épreuves orales de concours ${ }^{11}$ et ce serait forcer l'analyse que de voir dans leur respect l'intériorisation a priori par le candidat de l'ordre hiérarchique ou la manifestation de sa docilité.

Il est d'ailleurs à noter que tous les candidats que nous avons observés ont au moins partiellement intégré ces règles et il est probable que les cas de candidats se présentant à l'entretien en les transgressant ouvertement sont rares ${ }^{12}$. A l'inverse, certains candidats, souvent ADS, livrent aux examinateurs (consciemment - il s'agit alors d'une stratégie de présentation de soi - ou non - elle est dans ce cas le produit de dispositions acquises) une image d'eux-mêmes presque «militaire " qui se manifeste dans la rigidité de la posture, le contrôle et la raideur des mouvements et l'usage de marques appuyées de politesse. Cette présentation d'un soi (trop) discipliné les dessert : on leur suppose alors une inaptitude à la prise d'initiative, voire un manque de personnalité. L'entretien permet d'ailleurs assez mal de vérifier la capacité des candidats à prendre des décisions ou des initiatives. Les " mises en situation $\|^{13}$ pratiquées au cours de l'entretien ne peuvent en effet prétendre tester l'" autonomie ", ni le " courage " des candidats dans la mesure où ce qui, en situation réelle, pourrait nécessiter de mobiliser ces compétences, fait précisément défaut ici : exposition à une scène éprouvante, menace réelle ou supposée sur l'intégrité physique... Au mieux, elles éprouvent leur capacité à se projeter dans une situation fictive, à cerner les attentes des examinateurs et à élaborer une réponse sensée. Ajoutons que le répertoire de ces mises en situation est très limité. Il est donc assez aisé, notamment pour les candidats ADS, de s’y préparer.

Enfin, l'épreuve ne garantit en rien que la future recrue est respectueuse de la Loi et le sera dans l'exercice de ses fonctions. Au cours de l'entretien, le candidat est pourtant amené à se positionner par rapport à la Loi à l'occasion d'une mise en situation (qui revient dans quasiment tous les entretiens) ${ }^{14}$; on lui demande s'il verbaliserait un parent proche ou sa(son) compagne(on) mais, dans ce cas, une réponse négative est toujours préférée par le jury, en ce qu'elle révélerait l'" honnêteté " du candidat. Signalons, en outre, que nous n’avons jamais observé de mises en situation destinées à éprouver l'attachement des candidats aux valeurs républicaines et à l'État de droit.

11. Elles sont par exemple régulièrement rappelées dans les rapports de jury de concours de recrutement d'enseignants.

12. Les entretiens réalisés avec des candidats montrent que tous ont réfléchi, avant l'épreuve, à leur tenue vestimentaire, aux postures à adopter, certains allant même jusqu'à s'entraîner à s'asseoir, à rester sur une chaise sans faire de mouvements intempestifs...

13. Lors de l'entretien, les candidats sont fictivement placés dans une situation qu'ils sont susceptibles de renconter lors de l'exercice du métier et les jurés leur demandent d'expliquer ce que seraient leurs réactions. 14. Parmi les mises en situation les plus fréquemment utilisées figure le cas d'un véhicule de police en patrouille pris à partie par des jeunes dans une " cité ". 


\section{3. ... ou sanctionner la compétence interactionnelle}

Pour bénéficier d'un jugement positif, les candidats doivent donner des gages de "sérieux » : maîtrise de certaines connaissances qui témoigne d'un effort minimum de préparation, production d'un discours de justification recevable, soumission aux codes vestimentaires et de politesse usuels dans une situation d'entretien professionnel. Le jugement auquel s'exposent les rares candidats qui ne satisfont pas à ces exigences est sans appel : " touristes", "sont entrés parce quills ont vu de la lumière".

Cependant, on trouve, parmi les mal notés, fréquemment stigmatisés comme "mous ", des candidats qui invoquent pourtant une motivation légitime, qui sont capables de mobiliser des ressources cognitives et peuvent même, lorsqu'ils sont ADS, attester de compétences professionnelles reconnues par leur chef de service ${ }^{15}$.

C'est donc que l'épreuve se joue pour l'essentiel sur autre chose. Ces candidats mal notés présentent la particularité d'être mal à l'aise dans la situation d'entretien. Leur corps les trahit : rougissant, balbutiant, tremblant parfois, presque immobiles dans une posture très raide ou animés, au contraire, de mouvements répétés et non contrôlés, ils ne parviennent pas à masquer le "stress" auquel ils sont soumis. Ces manifestations corporelles ne sont pas rapportées par les examinateurs aux conditions de l'épreuve elle-même et ne sont pas interprétées comme le signe de difficultés des candidats à faire face à ce type de situation mais sont considérées comme des indicateurs substantiels de leur (manque de) personnalité et de leur faible motivation. Les résultats des tests psychométriques commentés par le psychologue sont alors souvent convoqués pour justifier l'appréciation, notamment lorsqu'ils pointent un "laxisme ", un "manque de rigueur", etc.

L'extrait suivant tiré de nos notes d'observation illustre parfaitement ces situations d'entretien au cours desquelles l'aisance dans l'interaction semble déterminante dans la formation du jugement, au détriment d'autres critères d'appréciation :

Le candidat est ADS ; il se présente pour la 7ème fois au concours de gardien de la paix. Cela devrait suffire à attester de sa motivation. Il est par ailleurs assez bien noté par son responsable et délivre un discours cohérent et recevable (au regard de ceux que nous avons pu entendre auparavant) sur les raisons de sa volonté d'être engagé dans la police. Mais il est très nerveux, ce qui se manifeste par un visage rouge et une posture très rigide. Il obtient la note de 7/20, justifiée par son "manque de motivation" et "de personnalité ". (Concours interne)

Les candidats jugés " moyens " se distinguent des précédents par le seul fait d'être un peu plus maitres de leurs émotions, tout en manifestant néanmoins une nervosité que laissent deviner la rigidité de leur posture ou leur difficulté à communiquer de manière aisée et fluide. Ils parviennent à convaincre le jury de leur " bonne volonté ", particuliè-

15. Dans le cas du concours interne, le jury dispose d'un rapport sur la «manière de servir » du candidat en tant qu'ADS, rédigé par son chef de service. 
rement lorsque leur situation sociale est jugée difficile et que leur est reconnu un certain "mérite ", mais ne suscitent aucun enthousiasme. Ils sont souvent ramenés à leur origine sociale populaire, qui apparaît dans ce cas comme un handicap. Un lien implicite est en effet établi par les examinateurs entre leur origine et un manque supposé d'autonomie et d'initiative, comme dans cet exemple :

Le candidat est ADS dans une compagnie républicaine de sécurité (CRS). Il est très tendu et répond par des phrases très courtes. Lors de la délibération, il s'attire les commentaires suivants: "Il est un peu lisse, ce n'est pas le mauvais bougre", "Il s'est coulé dans le moule CRS : c'est propre aux gars de la campagne, ils ont un truc, ils sy trouvent bien et ils ont presque peur d'aller voir ailleurs ", "Lui, s’il n'est pas encadré, il va avoir du mal à se diriger ", "Mais c'est quelqu'un de très droit, il va obéir». Il obtient 9/20. (Concours interne)

Pourtant, une origine populaire ne constitue pas en soi un handicap. Beaucoup de candidats issus de milieux populaires ${ }^{16}$ passent l'épreuve avec succès, tandis que l'on a pu observer des candidats issus de milieux sociaux plus favorisés faisant l'objet d'une très mauvaise appréciation.

Ce qui distingue des précédents les candidats biens notés, c’est précisément leur capacité à se comporter avec aisance dans l'interaction, sans sortir du cadre des comportements admissibles mais sans paraître devoir faire d'effort particulier pour s'y tenir. Ils parviennent à ne pas montrer qu'ils sont mis à l'épreuve, en dominant leurs émotions et en contrôlant leur corps. Ils apparaissent relativement spontanés, sont souriants et parviennent parfois même à plaisanter. Alors que la situation aurait pu apparaître comme instituant un «marché linguistique " particulièrement "tendu ${ }^{17}$ (Bourdieu 1982), une forme de franc-parler, de bagou, d'éloquence sans manière, est au contraire appréciée, quitte à ce que les normes lexicales, syntaxiques ou grammaticales les plus élevées soient transgressées. Ces candidats projettent alors une image d'eux-mêmes qui les fait apparaître cordiaux, aimables, "sympathiques" et - c'est important - comme "ne faisant pas de manière ". Le jugement des jurés semble alors entièrement informé par cette «impression», au détriment de tout autre critère d'appréciation.

16. A la session de février 2005, dernière session pour laquelle l'information a été saisie par l'administration, on pouvait compter, parmi les admis, au moins $22,3 \%$ d'enfants d'ouvriers et d'employés contre 4,8\% d'enfants de cadres supérieurs. L'imprécision de ces données doit néanmoins être soulignée : d'abord parce que les catégories de codage employées par l'administration de la Police nationale ne sont pas parfaitement conformes aux catégories les plus usuelles du sociologue ; ensuite parce que la catégorie " enfants de retraités " rassemble plus de $20 \%$ de notre population sans qu'il soit possible d'en savoir plus sur leur origine socioprofessionnelle.

17. Au sens où le contexte de l'interaction est très officiel et présente des enjeux importants pour les candidats. 
C'est clairement ce que montrent les deux extraits suivants :

Le commissaire membre du jury est sorti. Il revient après avoir aperçu le candidat qui doit être reçu : "Vous le verriez! Il doit être à Auchan lui aussi (le candidat précédent était salarié de ce groupe) mais au rayon boucherie!". On fait entrer le candidat. La remarque du commissaire s'explique sans doute par son physique "rond " : il a, comme on dit, une "bonne bouille ". L'entretien révèle l'aisance du candidat, son "bagou ", même si certaines des questions qui lui sont posées restent sans réponse. "Il est courageux et il est sympa en plus", "Alors, il aurait pu être boucher, hein? Il aurait pu tout faire ce gars-là!». Il se voit attribuer la note de 16/20. (Concours externe)

Le candidat est ADS à la Police de l'air et des frontières mais se présente ici au concours externe. On lui demande s'il connaît Benazir Bhutto ${ }^{18}$. Le candidat est très hésitant. Il confond le Pakistan avec l'Afghanistan et Benazir Bhutto avec (semble-t-il) Taslima Nasreen. Plus tard, lorsqu'on lui demande où est située la ville de Schengen, il répond (comme si l'examinateur lui avait demandé son chemin) : "Désolé, je ne peux pas vous renseigner ". Pendant tout l'entretien, il est souriant et paraît à son aise. Au moment des délibérations, qui durent quelques secondes, le commissaire s'exclame : "Il est bien!". "Même très bien » surenchérit le lieutenant. "Allez : 16!" annonce le commissaire. C’est effectivement la note qu'il obtient. (Concours externe)

C'est ainsi que, pour ces candidats dotés de ce que nous proposons d'appeler une forme de compétence interactionnelle (voir encadré 3), l'absence de réponse ou des réponses erronées aux questions de " culture générale " (pourtant jugée essentielle par la plupart des jurés) ne sont pas rédhibitoires alors qu'elles sont mobilisées comme éléments "à charge " pour les candidats moins à l'aise.

Par ailleurs, quand le candidat a fait bonne impression, le bilan des tests psychologiques n'est jamais évoqué au cours de la délibération, y compris lorsqu'il comportait des "éléments de personnalité " jugés problématiques chez d'autres. Il ne conduit donc pas à modifier ni même à nuancer le jugement. Ceci en dit long sur le statut réel accordé à ces tests, bien que de nombreux examinateurs déclarent les considérer comme un élément essentiel du dispositif, censé procéder au filtrage des candidats les moins aptes psychologiquement. Ils servent moins à informer le jugement qu’à légitimer ex post une appréciation négative.

18 Nous sommes à l'époque de son retour au Pakistan ; elle est alors en campagne pour les élections législatives.. 


\section{Encadré 3}

\section{La compétence interactionnelle : quelques pistes d'analyse}

L'épreuve d'entretien mobilise des stratégies, par lesquelles le candidat tente de contrôler consciemment l'impression qu'il produit. Mais, dans une interaction fortement asymétrique et porteuse d'enjeux importants, dont le déroulement ne peut être ni prévu ni cadré par le candidat, l'image de soi que l'individu projette n'est pas que le résultat d'une stratégie, comme en témoignent toutes les manifestations corporelles involontaires et incontrôlées (rougissements, balbutiements...) observables chez certains d'entre eux. L'aisance dont d'autres témoignent dans une telle situation n'est donc que marginalement le résultat d'une décision. Par ailleurs, si elle «émerge » (Eymard-Duvernay et Marchal, 1997) dans l'interaction, il nous semble difficile d'admettre que cette compétence interactionnelle soit produite par l'interaction, dont les conditions ne changent guère d'un candidat à l'autre. Elle nous semble devoir être appréhendée comme une « espèce » particulière de capital.

Nous pourrions parler d'une forme de "capital social incorporé », constitué de dispositions à interagir qui s'actualisent dans les manières d'être à un autrui non familier, dans le cadre d'interactions suffisamment brèves pour ne pas nécessiter de (trop) se dévoiler socialement. Elles incluent la maîtrise des codes sociaux régissant les différents types d'interactions (e.g. la connaissance des usages dans un entretien de type professionnel), qui permet d'éviter les " erreurs de cadrage » (i.e. les comportements inadaptés à un contexte particulier et mal défini par un des interactants) (Goffman, 1991), mais ne s'y réduisent pas. Elles permettent également de se mouvoir avec aisance, sans timidité excessive, et de parvenir à paraître " aimable » et sympathique, dans un cadre imposé ou négocié.

Le processus social au cours duquel se forme et s'accumule ce capital requiert sans doute une analyse fine - dépassant largement le cadre de cet article - de l'ensemble des interactions et des expériences socialisatrices qui ont cours dans le cercle familial et les groupes de pairs et qui contribuent à construire, dès la prime enfance, le rapport à un autrui non familier.

Si nous empruntons à la sociologie bourdieusienne le concept de capital, les propositions que nous formulons ici s'en éloignent quelque peu sous deux aspects (Bourdieu, 1982). D'abord, nous suggérons que ces dispositions à interagir sont non seulement largement transférables d'un contexte d'interactions à un autre (entretien de recrutement, conversation avec des inconnus...) mais aussi que leur efficacité (en termes de profit que peut retirer leur porteur du fait d'apparaître de prime abord sympathique à autrui) est - pour peu que la durée de l'interaction soit limitée dans le temps et exception faite des cas de trop grande distance culturelle entre interactants - en partie indépendante des propriétés sociales de ceux qui reçoivent le soi projeté. Elle ne se limiterait donc pas aux situations où la proximité sociale garantit une compatibilité des habitus de classe. 


\section{suite encadré 3}

Cette proposition permet de rendre compte du fait que la compétence interactionelle « fait impression » aussi bien sur un brigadier que sur un commissaire (voire sur le chercheur lui-même) $\left({ }^{*}\right)$, dont les positions et trajectoires sociales sont sensiblement différentes. Par ailleurs, la distribution de cette compétence dans l'espace social nous semble en partie obéir à des déterminants différents de ceux qui régissent la distribution d'autres ressources (économiques, culturelles ou linguistiques) et il n'est pas certain que les plus dominés culturellement ou économiquement en soient nécessairement dépourvus. Ainsi, nos observations montrent que des candidats d'origine populaire en sont dotés tandis que d'autres, de milieux sociaux plus favorisés, semblent dans l'incapacité d'activer ce type de dispositions.

$\left({ }^{*}\right)$ : En tant qu'observateur de la "scène ", nous étions, comme les examinateurs et bien qu'elle ne nous soit pas explicitement adressée, destinataire de la présentation de soi livrée par les candidats. Il est, de ce point de vue, remarquable que l'impression produite sur nous par les candidats (et formulée in petto) correspondait peu ou prou à celle dont faisaient état les examinateurs lors de la délibération.

L'importance décisive que revêt cette compétence interactionnelle appelle deux explications complémentaires l'une de l'autre.

D'abord, elle exerce sur les jurés un tel pouvoir de séduction, de " charme " (au sens fort du terme) ${ }^{19}$ qu'elle en occulte les éventuels "défauts " du candidat (absence de réponses ou réponses erronées aux questions de culture générale, résultats problématiques aux tests psychologiques par exemple). Mais elle semble également être implicitement interprétée par les jurés comme la manifestation d'une aptitude au travail en équipe (une dimensions constituante du travail des policiers subalternes») qui suppose notamment une capacité à participer à la sociabilité particulière ("virile ") qui se déroule dans les " coulisses " de la profession et à s'associer aux pratiques qui l'accompagnent (repas arrosés, plaisanteries à caractère sexuel ou scatologique...) (Pruvost, 2008). Cette explication permet de comprendre que les candidats issus de milieux sociaux favorisés ne soient pas systématiquement privilégiés par rapport aux candidats d'origine plus modeste. La prestation de ce candidat et l'appréciation qu'elle suscite illustrent bien cette idée :

Le candidat (concours interne) est ADS, bien noté par son chef de service. Son père est psychiatre, sa mère infirmière. Il explique avoir décidé de mettre fin à sa scolarité en fin de classe de seconde, après avoir constaté son peu d'intérêt pour les études. Il dit être devenu ADS par "vocation ", au terme d' "un choix mûrement réfléchi ». Tout au long de l'entretien, il se montre plutôt à l'aise et s'exprime dans une langue châtiée. Il répond correctement aux questions de «culture générale » comme aux questions plus professionnelles. Les

19. Le dictionnaire Le petit Larousse (édition 2002) définit notamment le " charme " comme un "attrait pystérieux exercé sur quelqu'un» et comme « ensorcellement »; 
jurés lui demandent ce que ses parents pensent de son choix d'intégrer la police (ce sera la seule fois que nous entendrons poser cette question) : il dit avoir été «soutenu» et " aidé». On lui demande : "Comment trouvez-vous vos collègues ADS ? Comment est l'ambiance avec eux? Le niveau, c'est pas toujours terrible, hein?" (là encore, ce type de question ne sera posé à aucun autre candidat observé). Il répond qu'il se sent très bien avec eux. Ce candidat obtient la note éliminatoire de $4 / 20$. Les jurés justifient cette note par les raisons suivantes : "Il est suffisant, condescendant ", "Il va user son environnement ", "Il manque d'humilité et de lucidité ». Ils lui attribuent des "difficultés d'intégration dans une équipe ». La prestation de ce candidat, conforme en de nombreux points aux attentes exprimées par les jurés, n’a pas réussi à inverser le cours d'un jeu qui semblait en grande partie perdu d'avance. Tout indique en effet que ce candidat a été victime de son origine sociale relativement élevée. Cette dernière semble, en effet, avoir été retenue contre lui, avant même le début de l'entretien, comme indice de son incapacité à travailler avec des collègues qui, en tendance, seront issus de milieux sociaux moins favorisés. Ce préjugé - au sens le plus strict ici - a été en quelque sorte confirmé par les attitudes et la manière de s'exprimer du candidat (jugé "condescendant", "suffisant»), qui n'ont fait que renforcer les jurés dans leur conviction qu'il ne pourrait s'intégrer à une équipe de gardiens de la paix et participer aux " activités de relâchement " pratiquées en coulisse, ces dernières, productrices de solidarité, relevant quasiment d'une nécessité fonctionnelle (Pruvost 2008).

D'une certaine manière, cette explication contribue également à nuancer quelque peu l'idée selon laquelle l'épreuve de l'entretien n'entretiendrait qu'un lien très lâche avec les exigences de la profession de policier.

\section{Conclusion}

L'épreuve d'entretien du concours de gardien de la paix est très partiellement standardisée et apparaît en tout point éloignée de l'enregistrement « objectif » d'une " compétence planifiée " (Eymard-Duvernay et Marchal, 1997). A ce titre, elle éloigne ce concours d'un mode de recrutement parfaitement bureaucratique, rationalisé et dépersonnalisé. Ce résultat peut sans doute être étendu à la plupart des recrutements dans la fonction publique dans la mesure où les prescriptions et les procédures officielles censées en définir les modalités requièrent toujours un travail d'interprétation par les recruteurs.

Par ailleurs, et contrairement à la perception qu'en ont les jurés pour qui c'est précisément sa fonction - ce qui en fait à leurs yeux un élément essentiel du dispositif de recrutement -, l'entretien ne permet guère d'éprouver la conformité des candidats aux exigences spécifiques du métier de policier (exigences dont les jurés proposent des définitions différentes). Certes, certaines ressources cognitives alliées à la capacité à verbaliser une "motivation " recevable sont indispensables, mais elles ne suffisent pas à garantir le recrutement des candidats qui en sont dotés. 
De plus, l'entretien permet mal de déceler l'existence de certaines (pré-) dispositions à la discipline ou au respect de la Loi.

De manière générale, et à la différence d'autres recrutements (dans les collectivités territoriales (Biland, 2008), à La Poste (Cartier, 2001), dans le travail social (Bodin, 2009)), il ne semble pas en mesure de garantir l'adhésion des futures recrues à l'institution ni même leur docilité qui laisserait supposer que les conditions d'une adhésion ultérieure sont remplies. Paradoxalement, même ceux qui sont en mesure d'offrir des gages solides de leur conformité à certaines attentes de l'institution (c'est le cas des Adjoints de Sécurité contractuels ADS - qui bénéficient d'appréciations favorables de leur chef de service) ne sont pas assurés d'être bien notés. En effet, la réussite à cette épreuve se joue pour l'essentiel sur ce que nous proposons de nommer la compétence interactionnelle qui permet aux candidats qui en sont le mieux dotés de faire bonne impression : à la maitrise des codes sociaux régissant ce type d'échanges (tenue vestimentaire appropriée, usage des formules de politesse attendues), ces candidats ajoutent une aisance dans l'interaction qui leur permet de masquer le fait qu'ils sont mis à l'épreuve (en dominant leurs émotions et en contrôlant leur corps) et de proposer une image d'eux-mêmes les faisant apparaître comme aimables et sympathiques. L'image ainsi projetée semble prendre le pas sur tout autre élément d'appréciation et informer complètement (et sans doute en partie inconsciemment) le jugement des jurés, jugement unanime malgré les attentes différenciées que ces derniers formulent et qui restent en partie «théoriques».

Finalement, et à l'exception notable du caractère collectif du procès de travail (la compétence interactionnelle semble en effet être interprétée par les jurés comme le signe d'une aptitude au travail en équipe), le mode de recrutement des gardiens de la paix semble donc s'inscrire dans un rapport relativement distendu aux caractéristiques et exigences spécifiques du métier. Cette proposition, qui pourrait sans doute et dans une certaine mesure être étendue aux modalités d'accès à d'autres emplois, ne doit pas, pour autant, conduire à penser le concours comme une phase inaugurale (après laquelle tout resterait à faire) du processus de "fabrication " des policiers. Les entretiens que nous menons actuellement auprès de candidats montrent, en effet, que beaucoup d'entre eux, y compris les " externes " qui, au contraire des ADS, ne sont pas déjà entrés dans l'institution, «s'y voient déjà » et témoignent par là d'une forme d'adhésion à l'institution, d'une "vocation " policière déjà en partie formée, comme s'ils étaient déjà entrés dans la "carrière morale ${ }^{20}$ " (Goffman, 1968) du policier. Il conviendrait alors d'examiner les conditions dans lesquelles peut être produite cette "vocation " policière et interroger l'existence de formes de socialisation anticipatrice (Van Maanen, 1973). Une telle analyse, couplée à une sociographie fine des candidats et des recrues et à l'étude approfondie de ce qui se joue dans les écoles de police (Cassan, 2005 ; Moreau de Bellaing, 2006 ; Pruvost, 2007), per-

20. Entendue ici comme le processus de construction de l'individu comme policier, qui l'amène à se vivre lui-même comme policier. 
mettrait de rendre compte des propriétés, dispositions et trajectoires sociales des (futurs) gardiens de la paix. Elle serait susceptible d'apporter une contribution à une meilleure compréhension de l'action de la Police nationale, jusque dans les distorsions possibles entre les objectifs qui lui sont fixés par le pouvoir et les objectifs réellement poursuivis par ses agents, entre ses effets attendus et ses effets réels.

\section{Bibliographie}

Arborio A-M., Fournier P. (2008), L'observation directe, Paris, Armand Colin.

Barbot J. (2010), «Mener un entretien de face à face » in Paugam S. (dir), L'enquête sociologique, Paris, PUF.

Becker H.S. (1985), Outsiders. Études de sociologie de la déviance, Paris, Métailié.

Biland E (2008), Concours territoriaux et institutionnalisation de l'emploi public local, Thèse sous la direction de Offerlé M., EHESS.

Biland E. (2010), "Carrières concourantes, éléments sur la socialisation par les concours administratifs territoriaux ", Terrains et travaux, $\mathrm{n}^{\circ} 17$.

Biland E. (2010), « Les ambiguïtés de la sélection par concours dans la fonction publique territoriale : une institutionnalisation inachevée ", Sociologie du travail, Avril-juin, vol. $52, \mathrm{n}^{\circ} 2$.

Biland E. (2010), «Les cultures d'institution » in Lagroye J. et Offerlé M. (dir.), (2010), Sociologie de l'institution, Paris, Belin.

Bittner E. (2001), "Florence Nightingale à la poursuite de Willie Sutton », Déviance et Société $25, \mathrm{n}^{\circ} 3$ pp. 285-305.

Bodin R. (2009), « Les signes de l'élection, Repérer et vérifier la conformation des dispositions personnelles des élèves éducateurs spécialisés ", Actes de la Recherche en Sciences Sociales, $\mathrm{n}^{\circ} 178$, pp. 80-87.

Boltanski L., Thévenot L. (1991), De la justification : Les économies de la grandeur, Paris, Gallimard.

Bourdieu P. (1982), Ce que parler veut dire, L'économie des échanges linguistiques, Paris, Fayard.

Cartier M. (2001), « Nouvelles exigences dans les emplois d'exécution des services publics. L’observation des épreuves orales du concours ", Genèse, n 42, pp. 72-81.

Cassan D. (2005), Une comparaison internationale de l'apprentissage et de la socialisation des policiers en France et en Angleterre. Le gardien de la paix et le constable, Thèse de doctorat sous la direction de Demailly L. et Duprez D., université de Lille 1. 
Darmon M. (2012), "Sélectionner, élire, prédire : le recrutement des classes préparatoires ", Sociétés contemporaines, n 86 , pp. 25-29.

Eymard-Duvernay F. et Marchal E. (1997), Façons de recruter : Le Jugement des compétences sur le marché du travail, Paris, Métailié.

Eymeri J.-M. (2001), La fabrique des énarques, Paris, Economica.

Goffman E. (1968), Asiles. Études sur la condition sociale des malades mentaux, Paris, Minuit.

Goffman E. (1973), La mise en scène de la vie quotidienne, 1. La présentation de soi, Paris, Minuit.

Goffman E. (1991), Les cadres de l'expérience, Paris, Minuit.

Gorgeon C. (1996), "Socialisation professionnelle des policiers : le rôle de l'école ", Criminologie, XXIX. 2, pp. 141-152.

Lagroye J. et Offerlé M. (dir.) (2010), Sociologie de l'institution, Paris, Belin.

Loubet del Bayle J.-L. (2006), Police et politique. Une approche sociologique, Paris, L'Harmattan.

Monjardet D. et Gorgeon C. (1993), La socialisation professionnelle des policiers, Étude longitudinale de la 121ème promotion des élèves gardiens de la paix. Tome 1, La formation initiale, Paris, Institut des Haute Études de la Sécurité Intérieure.

Monjardet D. (1996), Ce que fait la police. Sociologie de la force publique, Paris, La Découverte.

Monjardet D. (2005), "Gibier de recherche, la police et le projet de connaître ", Criminologie, vol. 38, ${ }^{\circ}$ 2, pp. 13-37.

Moreau de Bellaing C. (2006), La police dans l'État de droit, Les dispositifs de formation initiale et de contrôle interne de la police nationale dans la France contemporaine, Thèse de doctorat, dir. P. Favre, Paris, IEP.

Pruvost G. (2007), Profession : policier, Sexe : féminin, Paris, Ed. de la MSH.

Pruvost G. (2008), «Ordre et désordre dans les coulisses d'une profession. L'exemple de la police nationale ", Sociétés contemporaines, 2008/4, n 72, pp. 81-101.

Réau B. (2009), "Les modalités de l'embauche dans une multinationale de loisirs ", Actes de la Recherche en Sciences Sociales, $\mathrm{n}^{\circ}$ 178, pp. 100-113.

Skolnik J. (1975), Justice without Trial, New York, John Wiley (1ère éd. 1966)

Van Maanen J. (1973), "Observations on the Making of policemen", Human Organisations, 32 (4), pp. 407-18.

Weber M (1995), Économie et société, tome 1, «Les catégories de la sociologie», Paris, Pocket. 